Background and Objectives Our recent experiments have suggested that similarly to SLE, patients with primary Sjögren's syndrome (SS) manifest significantly increased phagocytosis of necrotic cell debris (secondary necrotic cell material, SNEC). This phenomenon has been attributed to serological aberrations of these patients, as indicated by the capacity of patients' sera to promote the uptake of SNEC by healthy phagocytes. In this study, we comparatively investigated the role of serum DNAse-I activity and IgG immunoglobulins from SS, SLE and RA patients in the promotion of SNEC-phagocytosis by healthy monocytes.

Materials and Methods The activity of DNase-I was assessed by single radial enzyme-diffusion assay (SRED) in the serum of patients with SS $(n=60)$, SLE $(n=22)$ and RA $(n=14)$ and healthy donors $(\mathrm{HBD}, \mathrm{n}=52)$. Total IgG immunoglobulins were isolated by negative selection from the serum of patients and controls using Melon Gel Resin columns. SNEC were prepared by heat-induced necrosis of normal lymphocytes and labelling with propidium iodide. The influence of serum components on SNEC-phagocytosis was assessed by flow cytometry in admixture experiments using normal phagocytes and SNEC pre-incubated with whole sera or purified serum IgG from patients or HBD.

Results Serum DNase-I activity in patients with SS and SLE was found significantly reduced compared to HBD and RA patients $(p<0.0001)$ and correlated inversely with the ability of these sera to promote SNEC-phagocytosis by healthy monocytes $(p=0.0003)$. The capacity of HBD sera to promote SNEC-phagocytosis by normal monocytes was significantly increased (by $90 \%$ ) following the addition of the DNase-I-specific inhibitor G-actin $(800 \mu \mathrm{g} / \mathrm{ml})$, supporting the important physiological role of DNA degradation by serum DNase-I in the prevention of SNEC-phagocytosis. SNEC opsonised with $\operatorname{IgG}$ isolated from autoimmune patients or from HBD were found to be similarly ingested by normal monocytes. However, in the presence of normal serum, the opsonisation of SNEC with IgG isolated from SS or SLE sera was found to induce significantly increased SNEC-phagocytosis, compared to that observed with SNEC opsonised with IgG isolated from HBD sera $(\mathrm{p}=0.001)$

Conclusions Our results indicate that, in a manner similar to SLE, SS patients are characterised by deficient serum DNase-I activity. Such reduced serum capacity for degradation of nucleic acids, in conjunction with the opsonisation of SNEC by serum autoantibodies appears to lead to increased exposure of the immune system of these patients to necrotic cell debris, to enhanced SNECphagocytosis and consequently to the inflammatory responses that characterise the disorder.

\section{A2.11 INVOLVEMENT OF THE NUCLEIC ACID RECOGNISING TOLL-LIKE RECEPTORS TLR7 AND TLR9 IN THE PATHOGENESIS OF EROSIVE ARTHRITIS}

doi:10.1136/annrheumdis-2013-203215.11

'Anita Fischer, 'Christina Böhm, 'Victoria Saferding, 'Eliana Goncalves-Alves, 2Marije Koenders, ${ }^{2}$ Wim van den Berg, 'Sonja Herman, 'Günter Steiner. 'Division of Rheumatology, Internal Medicine III, Medical University of Vienna, Austria; '2Department of Rheumatology, Radboud University Nijmegen Medical Centre, Netherlands

Background There is substantial evidence that abundant release and/or insufficient removal of endogenous nucleic acids can trigger autoimmune reactions via activation of nucleic acid recognising Tolllike receptors (TLR) such as TLR7 and TLR9, which may lead to the development of SLE and other systemic autoimmune diseases. However, in RA the involvement of these TLRs is less well understood. Interestingly, in rats with pristane-induced arthritis (PIA) disease can be transferred by $T$ cells together with antigen-presenting-cells pre-activated with TLR7 or TLR9 agonists (Hoffmann et al, J Autoimmunity 2011; 36: 288-300).
Objective We aimed to study the role of TLR7 and TLR9 in the pathogenesis of inflammatory erosive arthritis by antagonising them in PIA and the KRN serum transfer model.

Methods Arthritis was induced in rats with the mineral oil pristane, and in $\mathrm{C} 57 \mathrm{Bl} / 6$ mice by injection of KRN serum. Immunoregulatory oligodeoxynucleotide (ODN) sequences (IRS) antagonising TLR7 or TLR9 were applied either subcutaneously (PIA) or intra-peritoneally (KRN). A non-inhibitory ODN was used as control and PBS served as placebo. Arthritis was scored using established scoring systems, inflammation and bone erosion were quantitatively analysed by histology. Serum and cell culture cytokine levels were measured by ELISA.

Results While the TLR7 inhibitor and the control ODN showed no effect on arthritis development and severity, the TLR9 antagonist reduced arthritis severity significantly in PIA. Bone erosion was almost completely abolished, whereas it was moderately aggravated in animals treated with the TLR7 inhibitor. Furthermore, IL-6 serum levels were significantly reduced in animals treated with the TLR9 antagonist. However, these beneficial effects were only observed when the inhibitor was applied before disease onset. Moreover, neither inhibitor affected arthritis onset and severity in the serum transfer model, which is independent of the adaptive immune system.

Summary and Conclusions Inhibition of TLR9 significantly reduced inflammation and bone erosion in PIA but not in the KRN serum transfer model that reflects the late effector phase of erosive arthritis. Therefore, these results suggest important involvement of the DNA (CpG) recognising TLR9 in the initiation of autoimmune arthritis whereas nucleic acid binding TLRs do not seem to play a major role in the later phases of the disease. Antagonizing TLR9 in human RA may only act beneficial in the earliest phase of the disease.

\section{A2.12 PHENOTYPE CHANGES OF BLOOD EOSINOPHILS REFLECT ACTIVITY AND SEVERITY IN SYSTEMIC SCLEROSIS}

\section{doi:10.1136/annrheumdis-2013-203215.12}

${ }^{1}$ Dirk M Wuttge, ${ }^{2}$ Annika Andreasson, ${ }^{3}$ Ellen Tufvesson, ${ }^{1}$ Agneta Scheja, 'Roger Hesselstrand, 'Lennart Truedsson. 'Department of Clinical Sciences, Lund, Section of Rheumatology; ' ${ }^{2}$ epartment of Laboratory Medicine, Section of Microbiology, Immunology and Glycobiology; ${ }^{3}$ Department of Clinical Sciences, Lund, Section of Respiratory Medicine and Allergology, Lund University, Lund, Sweden

Background and Objectives We investigated whether surface marker expression analysis of blood eosinophils may reflect disease activity in systemic sclerosis (SSc), since a role for eosinophils in the pathogenesis of SSc may be suggested from the observation of increased counts in bronchoalveolar lavage.

Materials and Methods By flow cytometer analysis, eosinophils in whole blood were identified in 32 consecutive untreated patients using surface marker CD16 and CD9. Surface expression of markers CD11b, CD44, CD48, CD54, CD81 and HLA-DR was measured. Data were related to clinical measurements of the disease activity.

Results An increased blood eosinophil population with low surface expression of CD9 was identified in patients with early SSc, i.e. a disease duration of $<2$ years, compared to patients with longer disease duration $(P=0.003)$ and controls $(P=0.029)$. CD81 expression was lower in SSc patients compared to healthy individuals $(P=0.003)$. In patients with early SSc, CD81 levels correlated inversely to degree of skin involvement $(r=-0.67 ; P=0.009)$. CD48 levels were increased in early SSc and were associated with an increased concentration of alveolar nitric oxide in these patients $(r=0.84 ; P<0.001)$. CD16 expression on blood eosinophils was also higher in patients with early disease and was associated with loss of nailfold capillaries $(r=-0.78 ; P<0.001)$. 